\title{
DINÁMICA DEL ENSAMBLAJE ALGAL EPIFÍTICO EN EL SISTEMA DE PLANOS INUNDABLES DE AYAPEL A TRAVÉS DEL PULSO DE INUNDACIÓN
}

\section{DYNAMICS OF EPIPHYTIC ALGAE ASSEMBLAGE IN AYAPEL FLOOD PLAIN THROUGH FLOOD PULSE}

\author{
Yimmy Montoya-Moreno', Néstor Aguirre ${ }^{2}$
}

${ }^{1}$ Biólogo, Especialista en Educación Ambiental. Magíster en Biología. Doctor en Ingeniería Grupo Geolimna. Facultad de Ingeniería, Universidad de Antioquia, A. A. 1226, Medellín (Antioquia), Colombia; yimmymontoya3@hotmail.com; ${ }^{2 B i o ́ l o g o, ~}$ Magíster en Biología. Doctor en Ciencias Naturales Grupo Geolimna. Facultad de Ingeniería, Universidad de Antioquia, A. A. 1226, Medellín (Antioquia), Colombia; naguirre@udea.edu.co

Rev. U.D.C.A Act. \& Div. Cient. 16(2): 491-500, Julio- Diciembre, 2013

\section{RESUMEN}

Se evaluaron las variaciones espaciales y temporales de los ensambles de algas epifíticas asociadas a raíces de macrófitas, en 14 sitios de muestreo: siete zonas con condiciones lenticas y siete con condiciones lóticas del sistema de planos inundables de Ayapel. Se determinó que el grado de conectividad entre el río, los caños y las ciénagas aumentó en aguas altas, lo que produjo la uniformidad de las condiciones fisicoquímicas y limitó el desarrollo de condiciones locales. Así, los cuerpos más aislados alcanzan a generar un mayor grado de independencia del cuerpo principal, debido a la formación de gradientes horizontales. Esta individualización de las condiciones fisicoquímicas, se hace más evidente en la fase de aguas bajas. Se encontró variación de la diversidad y de la riqueza de especies a nivel espacial, ya que se presentó una baja similaridad florística entre los ensamblajes ficoperifíticos (30\%), lo que favoreció la riqueza alta de especies registrada. Las diatomeas predominaron en el sistema, siendo favorecidas por la concentración de amonio y, su abundancia, se redujo con el aumento en las precipitaciones. Las clorofíceas son favorecidas por el aumento en las precipitaciones, en la variación del nivel de la ciénaga, en las concentraciones bajas de nitratos, de nitrógeno amoniacal y de la conductividad eléctrica. Se halló que las concentraciones bajas de nutrientes y la disminución de la precipitación favorecen el desarrollo de las cianofíceas. Finalmente, se consideró a las euglenofíceas, dinofíceas y crisofíceas como grupos algales minoritarios, dentro del ensamblaje epifítico.

Palabras clave: Epifiton, perifiton, ciénagas tropicales, Colombia.

\section{SUMMARY}

Spatial and temporal variations of the epiphytic algae assemblages associated with roots of macrophytes in 14 sampling sites, seven zones with lentic and seven with lotic conditions of the Ayapel flood plain was evaluated. It was determined that the degree of connectivity between the river, the streams and swamp increased in high waters which produced physico-chemical uniformity conditions and limited the development local conditions. Thus, the most isolated bodies reach a greater degree of independence from the main body due to the formation of horizontal gradients. This individualization of the physico-chemical conditions is most evident in the low water phase. Diversity and richness of species variation at the spatial level was found, as well as lower floristic similarity (30\%) between the periphytics assemblages which favored the recorded high species richness. Diatoms predominated in the system, being favored by ammonium concentration; their abundance decreased with precipitation increase. Chlorophyceae are favored by the precipitation increase, variation in water level, low concentrations of nitrates, nitrogen ammoniacal and electrical conductivity. Low concentrations of nutrients and the decrease of precipitation favor the development of the cyanobacteria. Finally, Euglenophyceae, Dynophyceae and Chrysophyceae are considered as minority algal groups within the epiphytic assembly.

Key words: Epiphyton, periphyton, tropical swamps, Colombia. 


\section{INTRODUCCIÓN}

En los ambientes de planos inundables existen pocas investigaciones sobre el perifiton en el país (Rivera-Rondón et al. 2010; Montoya-Moreno \& Aguirre, 2008; 2009; MontoyaMoreno \& Ramírez, 2009). En las planicies de inundación, las condiciones específicas de cada hábitat favorecen la existencia de una plasticidad morfológica de la vegetación acuática, en donde el tamaño, la forma y las comunidades de especies presentes se modifican, de acuerdo a un gradiente fisicoquímico (Murphy et al. 2003; Rodrigues et al. 2008). Los lagos de planos de inundación son colonizados por diferentes especies de macrofitas acuáticas y éstas, a su vez, por el perifiton; juntos son responsables de la elevada productividad de los ecosistemas acuáticos. Estos intervienen en varios procesos relevantes en la dinámica de los ecosistemas (Schwarzbold, 1990). Estas con condiciones favorecen la diversidad biológica alta y los altos niveles de producción pesquera, que permiten el sostenimiento de cientos de miles de habitantes de los planos inundables colombianos (Montoya-Moreno et al. 2011).

Las algas perifíticas son indicadoras biológicas del nivel trófico del agua, lo que muestra variación con los cambios en la calidad del agua y la dinámica del sistema (Junk et al. 1989). Las investigaciones en sistemas cenagosos se han concentrado en el fitoplancton y en las macrófitas, al evaluar los productores primarios; sin embargo, la contribución del perifiton a la producción primaria de ecosistemas someros es fundamental (Montoya-Moreno \& Aguirre, 2009), por lo que se hace necesario realizar investigaciones en este tipo de ecosistema.

Por otro lado, a pesar de la gran abundancia de las áreas de inundación a escala mundial, Goldsborough \& Robinson (1996) consideran que las investigaciones ficológicas se ha concentrado en otros sistemas, como lagos y ríos, de modo que la información sobre los ensamblajes algales en los ambientes de planos de inundación, en forma general, son bastante fragmentadas, particularmente, en las áreas tropicales y subárticas.

El predominio de los diferentes grupos de algas en un determinado ecosistema es función de las características dominantes en el medio (Felisberto et al. 2001). Los sistemas de planos inundables están sometidos a variaciones altas del nivel de la columna de agua, como respuesta a las fluctuaciones de las precipitaciones propias del ciclo hidrológico. Este conjunto de cambios debe ser internalizado por los organismos, por lo que el objetivo de este trabajo es identificar las características fisicoquímicas y ambientales, que controlan el ensamblaje y generar un modelo predictivo de fluctuación del ensamblaje en función del pulso de inundación.

\section{MATERIALES Y MÉTODOS}

El sistema cenagoso de Ayapel, se ubica en la jurisdicción del municipio de Ayapel, en el departamento de Córdoba, en la planicie atlántica del norte de Colombia. Forma parte del macrosistema de humedales y zonas anegables de la depresión Momposina. La cuenca de la ciénaga tiene un área de $1504 \mathrm{~km}^{2}$, situada entre 20 y 150msnm. El territorio, se ubica en la zona de bosque húmedo tropical, con temperaturas entre los 26 y $29^{\circ} \mathrm{C}$ (IGAC, 1986).

Se realizaron nueve muestreos, entre el 2006 y 2009, que abarcaron tres ciclos hidrológicos, bajo condiciones diferenciales del pulso de inundación, tales como mayo 2006-aguas en ascenso, julio 2006-aguas en ascenso, septiembre 2006-aguas altas, diciembre 2006-aguas en descenso, marzo 2007-aguas bajas, agosto 2007-aguas en ascenso, enero 2008-aguas altas, septiembre 2008-aguas en descenso y enero 2009-aguas en descenso. Detalles sobre la información morfométrica, fisicoquímica y ambiental, se puede consultar en Montoya-Moreno \& Aguirre, 2009; Montoya-Moreno \& Ramírez, 2009; Montoya-Moreno et al. 2011.

En los parches de macrofitas, se determinaron cuáles eran las especies más abundantes por inspección visual del borde del parche; se tomaron tres muestras de raíces por cada especie de planta; se evalúo la transparencia, la temperatura del agua, el oxígeno disuelto y su porcentaje de saturación, la conductividad eléctrica, el $\mathrm{pH}$, la profundidad total, la alcalinidad y la dureza y se tomaron muestras de 3L de agua, para la determinación en el laboratorio de los nutrientes, tales como nitrógeno amoniacal, nitratos, nitritos, fósforo soluble reactivo y sulfatos, evaluados según APHA (2005).

Las muestras fueron depositadas en frascos de plástico de $100 \mathrm{~mL}$, se fijaron con solución de lugol, hasta obtener una concentración final del $4 \%$. Las raíces recolectadas fueron llevadas al laboratorio biológico del grupo Gaia, para la determinación de las especies del ficoperifiton, con base en literatura especializada (Montoya-Moreno et al. 2011). El conteo, se realizó a partir del uso de cámaras de Segdwick-Rafter de $1 \mathrm{~mL}$ de capacidad, empleándose 50 campos aleatorios, que se determinaron al tener en cuenta curvas de saturación de especies y para calcular la densidad del fitoperifiton se utilizó la fórmula de Ross (1979).

La composición, la abundancia y la diversidad de la comunidad, se evaluó mediante curvas de importancia y se usaron los índices comunitarios. Se empleó la prueba de correlación lineal múltiple de Spearman (rs) y un análisis de regresión lineal simple, para evaluar el efecto de las diferentes variables físicas, químicas y ambientales, sobre los grupos algales componentes del ensamblaje. 


\section{RESULTADOS Y DISCUSIÓN}

El momento hidrológico en el que se realiza el muestreo es el factor principal en el comportamiento de las condiciones fisicoquímicas del sistema de planos inundables de Ayapel.
Incluso, se encontró que para una misma fase del pulso en ciclos sucesivos, se registran diferencias en la dinámica del sistema. Las características fisicoquímicas del agua del sistema de planos inundables de Ayapel están dadas por las temperaturas elevadas del agua, la baja transparencia, conductividad, oxígeno disuelto y $\mathrm{pH}$ neutro (Tabla 1).

Tabla 1. Valores de cada variable respecto al pulso de inundación.

\begin{tabular}{|c|c|c|c|c|c|c|c|c|c|c|c|c|c|c|c|}
\hline Variable/sistema & Pulso & 1 & 2 & 3 & 4 & 5 & 6 & 7 & 8 & 9 & 10 & 11 & 12 & 13 & 14 \\
\hline \multirow[b]{4}{*}{ Profundidad (m) } & A.A & 3,5 & 4,2 & 4,2 & 4,5 & 4,2 & 3,5 & 3 & 2 & 3,7 & 3,3 & 5 & 4,3 & 2,9 & 5 \\
\hline & A.B & 4,3 & 5,7 & 5,7 & 6,2 & 2,5 & 3 & 2,4 & 3,1 & 4,5 & 3,5 & 5 & 3,6 & 3,6 & 3,45 \\
\hline & A.B.a & 3,3 & 5,2 & 3,5 & 4,84 & 3 & 2,3 & 1,7 & 2,8 & 3,7 & 2,04 & 3,3 & 3 & 2,9 & 3,3 \\
\hline & A.A.d & 1,2 & 2,3 & 1,8 & 3 & 1,5 & 0,61 & 0,7 & 2,32 & 3 & 0,7 & 0,41 & 0,25 & 2,9 & 0,41 \\
\hline \multirow{4}{*}{$\begin{array}{l}\text { Transparencia } \\
\text { (m) }\end{array}$} & A.A & 0,45 & 0,57 & 0,57 & 0,23 & 0,57 & 0,15 & 0,33 & 0,1 & 0,65 & 0,21 & 0,5 & 0,67 & 0,32 & 0,5 \\
\hline & A.B & 1,03 & 1,06 & 1,06 & 0,6 & 0,7 & 0,6 & 0,35 & 0,37 & 0,57 & 0,4 & 0,41 & 0,27 & 0,27 & 0,4 \\
\hline & A.B.a & 0,9 & 1,27 & 0,91 & 0,65 & 0,96 & 0,65 & 0,3 & 0,45 & 1,3 & 0,23 & 0,38 & 1 & 0,3 & 0,38 \\
\hline & A.A.d & 0,32 & 0,35 & 0,24 & 0,26 & 0,12 & 0,08 & 0,23 & 0,28 & 0,54 & 0,3 & 0,15 & 0,05 & 0,3 & 0,15 \\
\hline \multirow{4}{*}{$\begin{array}{c}\text { Temperatura del } \\
\text { agua }\left({ }^{\circ} \mathrm{C}\right)\end{array}$} & A.A & 29,8 & 29,4 & 29,4 & 30,2 & 29,4 & 28,9 & 31,9 & 30,6 & 28,2 & 31,2 & 29 & 32 & 29,6 & 29 \\
\hline & A.B & 32,2 & 31,8 & 31,8 & 31,9 & 34 & 30,3 & 34,1 & 32,3 & 30,2 & 31,6 & 29,1 & 31,7 & 31,7 & 35,6 \\
\hline & A.B.a & 30,1 & 29,2 & 30,5 & 31,5 & 30,5 & 32,1 & 31,7 & 31,4 & 31,1 & 30,4 & 32,8 & 31,7 & 31,5 & 32,8 \\
\hline & A.A.d & 31,4 & 29,9 & 30,3 & 30,2 & 29,5 & 32,3 & 29,5 & 31 & 31 & 32,3 & 29,8 & 34,1 & 29,5 & 29,8 \\
\hline \multirow{4}{*}{$\begin{array}{l}\text { Oxígeno disuelto } \\
(\mathrm{mg} / \mathrm{l})\end{array}$} & A.A & 1,13 & 6,88 & 6,88 & 5 & 6,88 & 3 & 4,9 & 5,52 & 3,25 & 8,8 & 6,3 & 4,45 & 1,24 & 6,3 \\
\hline & A.B & 2,79 & 5,46 & 5,46 & 6,09 & 5,42 & 5,2 & 5,78 & 6,14 & 7,11 & 4,89 & 7,98 & 0,6 & 0,6 & 7,03 \\
\hline & A.B.a & 2,51 & 4,2 & 5,51 & 6,4 & 2,53 & 5 & 6 & 5,1 & 5,6 & 4,6 & 5,9 & 0,6 & 3,87 & 5,9 \\
\hline & A.A.d & 5,8 & 4,65 & 5,03 & 5,62 & 3,25 & 6,03 & 6,6 & 6,69 & 3,18 & 4,46 & 4,97 & 6,02 & 3,25 & 4,97 \\
\hline \multirow{4}{*}{$\begin{array}{c}\text { Saturación } \\
\text { de oxígeno } \\
(\mathrm{mg} / \mathrm{l})\end{array}$} & A.A & 14 & 83,8 & 65,1 & 93,9 & 41,2 & 53 & 65,1 & 82 & 65,7 & 67,6 & 82,2 & 37,5 & 16 & 82,2 \\
\hline & A.B & 39,9 & 77 & 77 & 93,9 & 76,5 & 71 & 82,7 & 83,5 & 102 & 67 & 104 & 8,3 & 8,3 & 101 \\
\hline & A.B.a & 33 & 83,8 & 65,1 & 93,9 & 33 & 64,7 & 80 & 82 & 65,7 & 67,6 & 65,8 & 8,3 & 21 & 79,4 \\
\hline & A.A.d & 79 & 62 & 68 & 74,6 & 42,8 & 83,4 & 87,6 & 90,1 & 43 & 62 & 68 & 92 & 29 & 68 \\
\hline \multirow{4}{*}{$\begin{array}{c}\mathrm{pH} \text { (unidades de } \\
\mathrm{pH})\end{array}$} & A.A & 6,75 & 6,15 & 6,15 & 6,48 & 6,15 & 6,41 & 6,6 & 7,32 & 6,49 & 6,57 & 7,25 & 6,52 & 6,25 & 7,25 \\
\hline & A.B & 6,9 & 6,15 & 6,15 & 6,65 & 6,58 & 6,99 & 6,95 & 7,02 & 6,3 & 6,51 & 6,9 & 6,98 & 6,98 & 7,25 \\
\hline & A.B.a & 6,8 & 6,2 & 6,5 & 6,8 & 6,39 & 7 & 6,9 & 7 & 7,3 & 6,4 & 8 & 6,98 & 5,3 & 8 \\
\hline & A.A.d & 7,01 & 7,39 & 6,9 & 6,54 & 5,7 & 6,28 & 7,25 & 7,73 & 6,5 & 6,05 & 6,38 & 0,29 & 5,7 & 6,38 \\
\hline \multirow{4}{*}{$\begin{array}{c}\text { Conductividad } \\
\text { eléctrica }(\mu \mathrm{S} / \mathrm{cm})\end{array}$} & A.A & 127 & 33,2 & 33,2 & 60,1 & 33,2 & 47,8 & 110 & 75,2 & 41 & 25,8 & 103 & 76,4 & 152 & 103 \\
\hline & A.B & 82 & 12,1 & 12,1 & 21,2 & 17,7 & 15,5 & 52,1 & 24,2 & 30 & 18,9 & 44,5 & 131 & 131 & 37,1 \\
\hline & A.B.a & 113 & 19,5 & 20,9 & 53,9 & 44,5 & 41 & 85,4 & 51,3 & 50,5 & 26,9 & 66,5 & 131 & 108 & 66,5 \\
\hline & A.A.d & 164 & 23 & 19,3 & 43,5 & 31,6 & 40,7 & 50,8 & 45,5 & 80,4 & 34,7 & 59,6 & 38 & 31,6 & 59,6 \\
\hline \multirow[b]{4}{*}{ Nitratos (mg/l) } & A.A & 0,9 & 0,9 & 0,9 & 0,9 & 0,9 & 0,8 & 1,6 & 0,9 & 0,9 & 0,9 & 2,1 & 0,9 & 0,9 & 2,1 \\
\hline & A.B & 0,8 & 2,4 & 2,4 & 4 & 1,6 & 4 & 4 & 4 & 0,9 & 0,9 & 1,19 & 0,06 & 0,06 & 4 \\
\hline & A.B.a & 4,6 & 0,9 & 1,06 & 0,12 & 4,2 & 0,1 & 1,6 & 0,12 & 0,19 & 0,15 & 1,4 & 0,46 & 0,5 & 1,4 \\
\hline & A.A.d & 1,3 & 1,8 & 1,06 & 2,6 & 0,5 & 5 & 4,2 & 2,8 & 0,8 & 1,5 & 4,5 & 0 & 1,4 & 4,5 \\
\hline \multirow[b]{4}{*}{ Nitritos (mg/l) } & A.A & 0,03 & 0,02 & 0,02 & 0,03 & 0,02 & 0,07 & 0,53 & 0,06 & 0,04 & 0,13 & 0,07 & 0,08 & 0,04 & 0,07 \\
\hline & A.B & 0 & 0 & 0 & 0,04 & 0,53 & 0,04 & 0,04 & 0,05 & 0,04 & 0,04 & 0,19 & 0,1 & 0,1 & 0,11 \\
\hline & A.B.a & 0,33 & 0,02 & 0,01 & 0,06 & 0,4 & 0,03 & 0,53 & 0,06 & 0,09 & 0,05 & 0,06 & 0,07 & 0,05 & 0,06 \\
\hline & A.A.d & 0,07 & 0,13 & 0,01 & 0,16 & 0,03 & 0,71 & 0,4 & 0,16 & 0,04 & 0,15 & 0,31 & 0 & 0,1 & 0,31 \\
\hline
\end{tabular}


Continuación Tabla 1.

\begin{tabular}{|c|c|c|c|c|c|c|c|c|c|c|c|c|c|c|c|}
\hline \multirow{4}{*}{$\begin{array}{l}\text { Nitrógeno amo- } \\
\text { niacal (mg/l) }\end{array}$} & A.A & 0,05 & 0,17 & 0,17 & 0,22 & 0,17 & 0,07 & 0,09 & 0,17 & 0,03 & 0,09 & 0,21 & 0,18 & 0,2 & 0,21 \\
\hline & A.B & 0,02 & 0,02 & 0,02 & 0,09 & 0,09 & 0,1 & 0,17 & 0,05 & 0,01 & 0,06 & 0,03 & 0,1 & 0,1 & 0,15 \\
\hline & A.B.a & 0,01 & 0,17 & 0,1 & 0,1 & 0,3 & 0,1 & 0,09 & 0,1 & 0,1 & 0,01 & 0,15 & 0,12 & 0,14 & 0,15 \\
\hline & A.A.d & 0,09 & 0,1 & 0,1 & 0,1 & 0,01 & 0,42 & 0,3 & 0,07 & 0,01 & 0,01 & 0,24 & 0 & 0,1 & 0,24 \\
\hline \multirow[b]{4}{*}{ Fosfatos (mg/l) } & A.A & 0,36 & 0,21 & 0,21 & 0,47 & 0,21 & 0,97 & 0,66 & 0,62 & 0,4 & 1,57 & 1,15 & 1,11 & 0,72 & 1,15 \\
\hline & A.B & 0,1 & 0,1 & 0,1 & 0,24 & 0,66 & 0,21 & 0,36 & 0,49 & 0,1 & 0,2 & 56 & 0,53 & 0,53 & 1,91 \\
\hline & A.B.a & 0,18 & 0,21 & 0,1 & 0,19 & 1,8 & 0,2 & 0,66 & 0,19 & 0,34 & 0,3 & 0,2 & 1,11 & 0,12 & 0,18 \\
\hline & A.A.d & 0,36 & 0,5 & 0,1 & 0,7 & 0,22 & 2,8 & 1,8 & 0,9 & 0,46 & 0,5 & 1,9 & 1,7 & 0,6 & 1,9 \\
\hline \multirow[b]{4}{*}{ Sulfatos (mg/l) } & A.A & 4 & 5,3 & 4 & 5,1 & 4 & 4 & 10 & 7 & 4 & 5,2 & 13 & 4 & 5,4 & 13 \\
\hline & A.B & 0,2 & 5 & 0,56 & 5,28 & 3,9 & 0,99 & 7,3 & 7 & 0,22 & 0,26 & 3,37 & 0,12 & 5,2 & 1,05 \\
\hline & A.B.a & 1,15 & 5 & 2,52 & 5,28 & 3,9 & 0,11 & 5 & 6,4 & 0,15 & 0,06 & 0,45 & 2,64 & 5 & 0,45 \\
\hline & A.A.d & 0,39 & 4 & 2,52 & 0,6 & 3,9 & 1,67 & 4 & 5 & 0,2 & 0,39 & 1,3 & 3 & 4 & 1,3 \\
\hline
\end{tabular}

Convenciones: AA: Aguas altas, AB: aguas bajas, ABa: aguas bajas en ascenso, AAd: aguas altas en descenso, 1) Ciénaga Playa Blanca, 2) Ciénaga Escobillitas, 3) Ciénaga Escobillas, 4) Ciénaga Ayapel frente al pueblo, 5) Ciénaga Hoyo los Bagres, 6) Ciénaga Paticos, 7) Ciénaga La Miel, 8) Caño Pinto, 9) Caño Barro, 10) Caño Quebradona, 11) Caño Grande, 12) Caño Muñoz Gamba, 13) Caño Muñoz Gusanera y 14) Caño Viloria.

Las variables fisicoquímicas, se pueden ordenar en tres grupos: las de baja variabilidad ( $\mathrm{CV}<15 \%)$, como la temperatura del agua y el $\mathrm{pH}$; las de variabilidad media $(40 \%>\mathrm{CV}<$ del $75 \%$ ), como la transparencia, la profundidad, el oxígeno disuelto y el porcentaje de saturación de $\mathrm{O}_{2}$, la conductividad eléctrica y el tercer grupo, de mayor variabilidad (CV> 90\%), que incluye los nutrientes (nitratos, nitritos, nitrógeno amoniacal, FSR y sulfatos) y los SDT.

El análisis de correlación lineal múltiple (Tabla 2) evidenció que son pocas las relaciones estadísticamente significativas halladas: 1) De manera positiva, se relacionó la alcalinidad con la abundancia de las diatomeas $\left(r^{2}=0,45 ; p=0,013\right)$; las dinofíceas con la concentración de sulfatos y la concentración de nitritos $\left(r^{2}=0,45 ; p=0,011 ; r^{2}=0,31, p=0,026\right)$; la transparencia del agua y el número total de algas perifíticas (abundancia total), las clorofíceas y la diversidad de especies $\left(r^{2}=0,5 ; p=0,001 ; r^{2}=0.44, p=0,015\right.$, respectivamente); 2) En forma negativa, se relacionó la alcalinidad con la abundancia de las clorofíceas $\left(r^{2}=-0,44 ; p=0,014\right)$, la abundancia de diatomeas y de cianofíceas con la diversidad $\left(r^{2}=-0,41\right.$; $p=0,021 ; r^{2}=-0.36 ; p=0.045$, respectivamente).

Se observó que hay variación de los estimadores de la composición, la abundancia y la diversidad del ensamblaje, a través del pulso y entre ambientes lénticos y lóticos, incluso, entre el grupo de ciénagas y de caños (Figura 1). Se identifica que no se presenta dominancia alta de ninguna morfoespecie, ya que las abundancias relativas de las especies no alcanzan a superar el $20 \%$. Se evidencian algunas tendencias, ya que la equitatividad muestra un valor medio poco variable durante toda la investigación; la dominancia revela pequeñas variaciones, en especial en aguas bajas en ascenso (mayo 2007) y presentó los mayores valores en aguas altas en des-

Tabla 2. Matriz de correlaciones entre las variables físicas y químicas.

\begin{tabular}{|c|c|c|c|c|c|c|c|c|c|c|c|}
\hline Variables & $\mathrm{N}-\mathrm{NH}_{4}$ & $\mathrm{~N}-\mathrm{NO}_{3}$ & $\mathrm{~N}-\mathrm{NO}_{2}$ & FSR & Zsd & D & OD & $\mathrm{pH}$ & C.E & T.w & Precip \\
\hline $\mathrm{N}-\mathrm{NH}_{4}$ & & 0,43 & 0,53 & 0,60 & $-0,37$ & $-0,23$ & 0,41 & 0,20 & 0,05 & 0,09 & $-0,39$ \\
\hline $\mathrm{N}-\mathrm{NO}_{3}$ & 0,43 & & 0,62 & 0,43 & $-0,29$ & $-0,26$ & 0,29 & 0,20 & $-0,06$ & 0,14 & $-0,28$ \\
\hline $\mathrm{N}-\mathrm{NO}_{2}$ & 0,53 & 0,62 & & 0,59 & $-0,32$ & $-0,51$ & 0,09 & 0,08 & 0,08 & $-0,01$ & $-0,19$ \\
\hline FSR & 0,6 & 0,43 & 0,59 & & $-0,54$ & $-0,44$ & 0,32 & $-0,2$ & $-0,01$ & 0,07 & $-0,29$ \\
\hline Zsd & $-0,37$ & $-0,29$ & $-0,32$ & $-0,54$ & & 0,66 & $-0,27$ & 0,14 & $-0,23$ & $-0,19$ & 0,29 \\
\hline $\mathrm{D}$ & $-0,23$ & $-0,26$ & $-0,51$ & $-0,44$ & 0,66 & & $-0,13$ & 0,27 & $-0,27$ & $-0,22$ & 0,20 \\
\hline OD & 0,41 & 0,29 & 0,09 & 0,32 & $-0,27$ & $-0,13$ & & 0,04 & $-0,28$ & 0,36 & $-0,31$ \\
\hline $\mathrm{pH}$ & 0,2 & 0,2 & 0,08 & $-0,16$ & 0,14 & 0,27 & 0,04 & & 0,13 & $-0,13$ & 0,01 \\
\hline C.E & 0,05 & $-0,06$ & 0,08 & $-0,01$ & $-0,23$ & $-0,27$ & $-0,28$ & 0,13 & & 0,02 & $-0,28$ \\
\hline T.w & 0,09 & 0,14 & $-0,01$ & 0,07 & $-0,19$ & $-0,22$ & 0,36 & $-0,1$ & 0,02 & & 0,17 \\
\hline Precipitac & $-0,39$ & $-0,28$ & $-0,19$ & $-0,29$ & 0,29 & 0,20 & $-0,31$ & 0,01 & $-0,28$ & 0,17 & \\
\hline
\end{tabular}


censo (2008), ambos momentos asociados al mismo año hidrológico. Finalmente, la diversidad registra una tendencia a aumentar en el tiempo, al igual que la riqueza de especies.
El nivel de la ciénaga estuvo relacionado, de manera negativa, con la concentración de nitratos y FSR y, en forma positiva, con el pH. Las variables físicas, químicas y climáticas
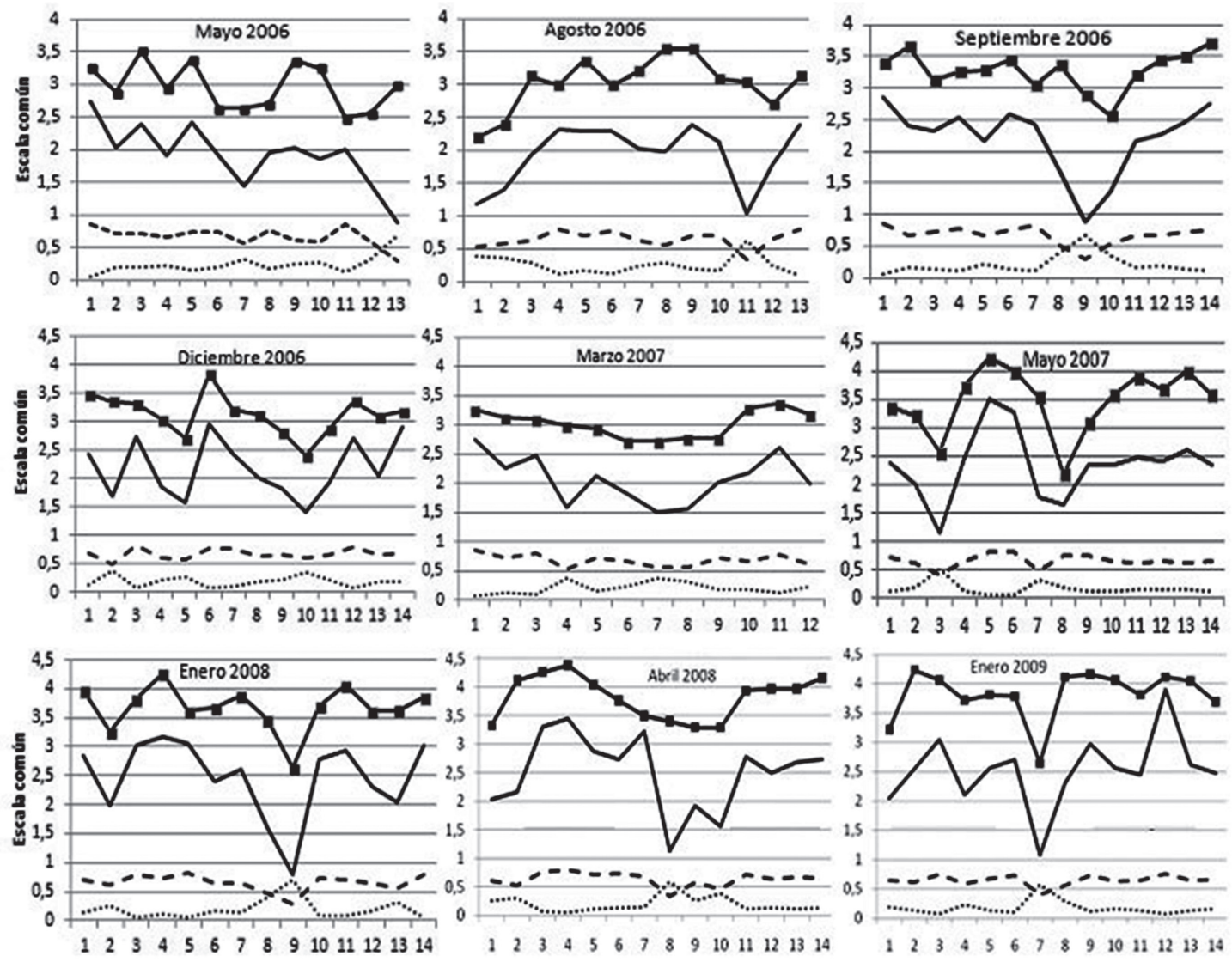

D $\longrightarrow \mathrm{H}^{\circ}---\mathrm{J} \rightarrow \ln S$

Figura 1. Variación espacial y temporal de los índices comunitarios en las 14 estaciones de muestreo. Simbología: D: dominancia; $\mathrm{H}^{\prime}$ : diversidad; J: equitatividad; S: riqueza de especies.

Estaciones de muestreo: 1) Ciénaga Playa Blanca, 2) Ciénaga Escobillitas, 3) Ciénaga Escobillas, 4) Ciénaga Ayapel frente al pueblo, 5) Ciénaga Hoyo los Bagres, 6) Ciénaga Paticos, 7) Ciénaga La Miel, 8) Caño Pinto, 9) Caño Barro, 10) Caño Quebradona, 11) Caño Grande, 12) Caño Muñoz Gamba, 13) Caño Muñoz Gusanera y 14) Caño Viloria.

evaluadas no registraron relación estadística significativa con los estimadores de diversidad, excepto la dureza (relación negativa con la riqueza de especies) y la concentración de nitritos (relación positiva con la riqueza de especies y con la abundancia total de algas en la matriz perifítica).

Las diatomeas registraron mayor abundancia en los periodos de aguas bajas, ya que, en esta situación, el sistema tiende a fragmentarse en varios subsistemas, estos quedan total o parcialmente aislados del cuerpo principal. Bajo estas circunstancias, la temperatura alta, la resuspensión del sedimento y la disminución de la transparencia generan condiciones limitantes para las poblaciones perifíticas (MontoyaMoreno et al. 2011).
Las diatomeas poseen diminutos tamaños que les permiten tener una rápida fijación de nutrientes en una menor superficie, por lo que son favorecidas en sistemas con velocidades de la corriente moderadas a altas (Horner et al. 1990). La mayoría de las diatomeas registradas en la investigación presentaron estructuras especializadas para la fijación al sustrato, como pedúnculos mucilaginosos en las especies del género Gomphonema, matrices gelatinosas, como en Cymbella, Navicula, Encyonema y colonias en forma de estrella o de ramos, como Eunotia, Fragillaria, Diadesmis. Este conjunto de características ofrecen ventajas competitivas al grupo de las diatomeas sobre las otras algas, razón por la cual, no solamente se encuentran estos organismos adheridos al sustrato o matriz perifítica sino que es posible 
y común, según el medio, encontrar a las diatomeas, como epífitos de otras algas, especialmente, de las formas filamentosas, como Oedogonium.

Respecto a las características hidráulicas, bajo las condiciones de aguas bajas el tiempo de retención hidráulico, se reduce, ya que disminuye el caudal. Numerosos investigadores han registrado que las diatomeas son favorecidas por caudales medios a altos (Allan, 1995; Stevenson, 1996; Montoya-Moreno \& Ramírez, 2007), los cuales, se presentan en la época de lluvias en este sistema. Incluso, se ha llegado a plantear que, en regiones con caudal medio, se presenta un mejor desarrollo del ensamblaje (Martínez \& Donato, 2003; Rivera-Rondón \& Díaz, 2004; Zapata \& Donato, 2005; Ramírez \& Plata, 2008).

Se encontró que el aumento de las precipitaciones, el nivel de la ciénaga y la conductividad eléctrica afectan el ensamblaje, mediante una reducción en la abundancia de las diatomeas. Otro aspecto a tener en cuenta es el efecto del material resuspendido durante las aguas bajas en la ciénaga, material que limita la transparencia del agua y, posiblemente, la fijación sobre las macrófitas, debido al efecto del oleaje, lo que redunda en la disminución de la densidad algal y el aumento de la dominancia de las diatomeas, que se ven favorecidas por tener estructuras especializadas para su fijación.

La resuspensión de sedimentos por acción del viento es uno de los principales factores que gobiernan el funcionamiento de los sistemas someros (Goldsborough \& Robinson, 1996), lo cual, parece, en el sistema de Ayapel, favorecer en aguas bajas a las diatomeas, pero limita la densidad algal epifítica y la diversidad total de la matriz. Conde et al. (1999), en lagunas de Uruguay, encontraron que el principal efecto del proceso de resuspensión es el aumento de la densidad y la biomasa algal en la columna de agua, así como de su productividad por unidad de área y eficiencia fotosintética; estas condiciones han sido registradas en la ciénaga de Ayapel en el plancton (Hernández-Atilano et al. 2005; Montoya-Moreno \& Aguirre, 2010). Se podría inferir que la mayor disponibilidad de energía y de turbulencia del agua exporta material del perifiton al plancton; además, que limita la adhesión del metafiton al perifiton, por lo que en el epifiton, se presentaría una condición inversa, es decir, la resuspensión, como un factor que discrimina qué organismos pueden conformar la biopelícula y limita la densidad que pueden alcanzar. La resuspensión inducida por el viento y por los animales puede afectar al cuerpo de agua, según su morfometría (Thomaz et al. 2007), ya que al aumentar el perímetro disminuye la pista de acción del viento sobre el espejo de agua.

Las algas clorofíceas, se vieron favorecidas por el aumento en las precipitaciones, en especial, sobresalen el grupo de las desmidiáceas, que alcanzan más del $90 \%$ de la riqueza grupal. De acuerdo con Coesel (1982), la composición y la abundancia de las desmidias está relacionada con los valores elevados de la temperatura del agua, del $\mathrm{pH}$, de la conductividad y de la mayor riqueza de macrofitas. Rodrigues \& Bicudo (2001) relacionaron la gran riqueza de desmidias perifíticas con la presencia de estanques de macrofitas. La presencia de las macrofitas estimula el desarrollo de las algas perifíticas al proporcionar el sustrato para la fijación y por el aporte de material nutritivo proveniente de su propio proceso de envejecimiento y muerte (Schwarzbold, 1990; Felisberto et al. 2001).

Otra característica que favorece a las desmidias es la alta temperatura promedio del agua, ya que las temperaturas entre 25 y $30^{\circ} \mathrm{C}$ son ideales para el desarrollo de estas algas, ya que su origen es tropical (Coesel, 1982).

La variación del nivel de la ciénaga y de las precipitaciones estuvo asociada con la abundancia de las clorofíceas, puesto que al aumentar la columna de agua acentuó la transparencia, lo que generó un incremento en la riqueza y abundancia de las clorofíceas, además de un aumento en la diversidad algal perifítica. Se ha considerado que las clorofíceas son fuertemente dependientes de una disponibilidad alta de nutrientes en el agua (Borchardt, 1996), por lo que los resultados aquí encontrados están de acuerdo con esta afirmación, ya que las clorofíceas mostraron mayores abundancias en aguas altas, aunque en el último pulso estudiado (20082009), se evidencia una tendencia opuesta.

Las concentraciones bajas de nitratos, de nitrógeno amoniacal y de los valores bajos de la conductividad eléctrica favorecen el predominio de las clorofíceas en el sistema cenagoso de Ayapel. Respecto a esta variable, Felisberto et al. (2001) encontraron que las desmidias presentaron una mayor abundancia en las zonas lenticas de un embalse brasilero, cuando aumentó la conductividad eléctrica, la temperatura del agua y el $\mathrm{pH}$.

Sobre el incremento en las densidades de desmidias perifíticas en aguas con bajas concentraciones de nutrientes, Coesel (1996) afirma que estas algas son encontradas, generalmente, en aguas ácidas, pobres en nutrientes y con baja conductividad eléctrica (valores inferiores a $100 \mu \mathrm{S} / \mathrm{cm}$ ), lo que explica el predominio que alcanza este grupo dentro del ensamblaje perifítico, en el sistema de Ayapel. Este último aspecto es cuestionable, puesto que las relaciones con las diatomeas son un factor a considerar, además de la mezcla, la cual, puede facilitar la liberación de desmidiáceas de la matriz o del metafiton hacia el plancton.

Las concentraciones altas de nutrientes, la disminución de las precipitaciones y niveles del agua intermedios y el aumento de la temperatura del agua favorecen el desarrollo de 
las cianobacterias. Roldán \& Ramírez (2008) consideran que estas abundan en los meses más calientes del año; condiciones similares fueron registradas por Huszar et al. (2000). Según Schindler (1977), una de las hipótesis de la posible causa del desarrollo de cianobacterias en sistemas acuáticos es la relación entre nitrógeno y fósforo. Cuando esta relación es menor que 10 , se espera el desarrollo de las cianobacterias. Condiciones de limitación de nutrientes, particularmente nitrógeno, benefician a las cianobacterias por: i) su habilidad para fijar nitrógeno molecular (formas fijadoras); ii) su capacidad de migrar a lo largo de la columna de agua, para tener acceso a los nutrientes (formas con pseudovacuolas o aerótopos) y, iii) su competencia exitosa, por el amonio frente a otras algas más grandes, por su alta relación superficievolumen, debido a sus formas picoplanctónicas (Wenzel \& Díaz, 2008).

Las cianobacterias presentaron baja abundancia y su distribución no alcanza mayores valores en la estructura del ensamblaje; por ser un sistema somero, la polimixis reduce sus densidades, aunque se alcanzan a presentar blooms de Cylindrospermopsis raciborskii en la columna de agua del sector de La Miel. Algunas cianobacterias son capaces de almacenar fósforo (Majewski-Algarte et al. 2006), lo que favorece el aumento de la densidad de ese grupo, incluso, en aguas altas, cuando la concentración de fósforo disminuye.

Las euglenofíceas, dinofíceas y crisofíceas son los grupos algales minoritarios dentro del ensamblaje epifítico en el sistema de Ayapel; se evidenció que las crisófitas exhibieron un mayor número de relaciones con variables abióticas y bióticas. Vale la pena ampliar la información sobre este grupo, ya que es escasa la información taxonómica y ecológica registrada en Colombia.

La densidad de crisofíceas aumenta con el incremento en la temperatura del agua, el $\mathrm{pH}$ y el nivel de la ciénaga y disminuye con el aumento en la concentración del PSR, la conductividad eléctrica y la diversidad de especies, en el sistema de planos inundables de Ayapel. Roldán \& Ramírez (2008) afirman que las crisofíceas, en general, se relacionan con aguas pobres en nutrientes y viven, en su mayoría, en aguas oligotróficas; estas relaciones se han encontrado también en el epifiton de la ciénaga de Ayapel.

Las euglenofíceas evidenciaron un ligero incremento en su abundancia en aguas bajas, pero respecto a otros grupos algales, se les consideró como acompañantes de los grupos mayoritarios (diatomeas, clorofíceas y cianoprocariotas), junto con las crisofíceas y los dinoflagelados. Una situación similar fue descrita por Donato (2001), quien estudió las variaciones en la composición del fitoplancton en algunas ciénagas del Magdalena, de acuerdo con los cambios hidrológicos en su ciclo anual; en la época de verano, debido a la disminución de nutrientes, se favorece la presencia de dinoflagelados, como Peridinium sp. y crisofíceas, como Dinobryon divergens, mientras que en la época de inundación, se observa el desarrollo de euglenofíceas.

Las dinofíceas fueron el único grupo que presentó relación positiva con la concentración de sulfatos; además, evidenciaron una relación inversa con la diversidad y el número total de organismos en el ensamblaje epifítico. Según Taniguchi et al. (2005), los fitoflagelados presentan picos de desarrollo asociados a los periodos posteriores a la perturbación (mezcla de la columna de agua por el viento o periodos de precipitación); es, en este momento, cuando tienen ventajas competitivas sobre los otros grupos de algas, por lo que son considerados como oportunistas.

Al comparar las ciénagas y los caños, se evidencia que existen diferencias entre las especies ficoperifíticas de mayor importancia, ya que en los caños dominan las diatomeas, mientras que en las ciénagas, este grupo comparte importancia con las clorofíceas y unas pocas cianofíceas y euglenofíceas. El aumento del tiempo de retención hidráulico en ambos sistemas, sumado a los procesos de estratificación térmica y química, inciden en la distribución de los organismos. Otro aspecto relevante tiene que ver con que algunas estructuras están predominantemente condicionadas por la potamofase, otras por la limnofase, en tanto que otros han sido favorecidos por su capacidad de adecuación a una amplia gama de condiciones del régimen pulsátil y se los conoce como euritípicos (Neiff, 1999).

La riqueza de especies fue mayor en los caños que en las ciénagas (350 contra 288), debido al aumento de la heterogeneidad espacial y de los gradientes térmicos y químicos en las ciénagas. Una situación similar fue encontrada por Rodrigues \& Bicudo (2001), quienes compararon la flora del perifiton en tres sistemas, con diferente régimen hidrodinámico, en un sistema de planos inundables brasileros. Estos autores concluyen que el tipo de ambiente y no el régimen hidrológico ejercieron mayor influencia sobre la riqueza específica de la comunidad. Otro aspecto a tener en cuenta es el aporte de algas desde los caños hacia las ciénagas, lo que incidiría en la mayor riqueza en estos ambientes dirigidos por el flujo. Finalmente, la ampliación registrada en los últimos años de la potamofase sobre la limnofase en el sistema, debería ser objeto de estudio de futuros trabajos.

El ficoperifiton mostró cambios en su estructura en relación al pulso de inundación. De esta forma, se pudieron discriminar cuatro ensambles característicos de los diferentes momentos hidrológicos ocurridos en el sistema (Figura 2).

1) Aguas Bajas. Aumento del efecto de cada sistema (caños y ciénagas) sobre el ensamble, debido a la reducción del 


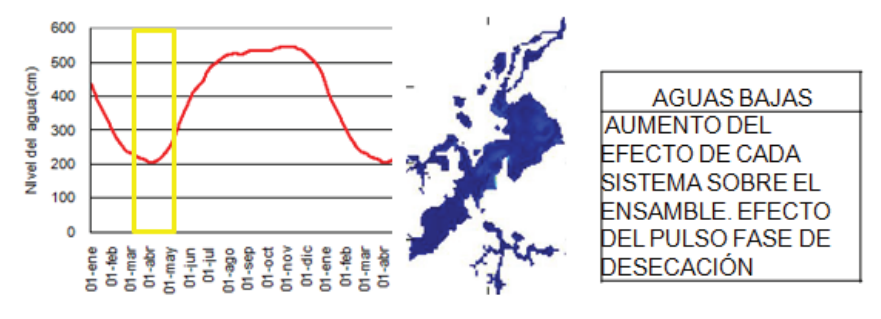

\begin{tabular}{l}
\hline \multicolumn{1}{|c|}{ AGUAS BAJAS } \\
\hline CIENAGAS \\
PREDOMINIO DE LAS ESPECIES Planothidium lanceolata, \\
Encyonema jetlandicum Y Oscillatoria sp1. \\
CAÑOS \\
PREDOMINIO DE LAS ESPECIES Navicula aff cryptocephala, \\
Stenopterobia delicatissima Y Eunotía naegueli \\
\hline
\end{tabular}
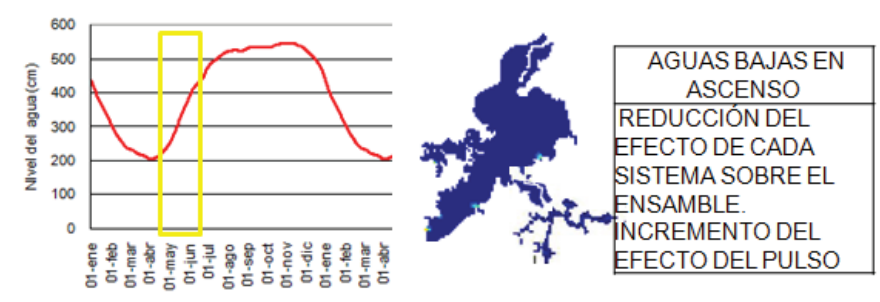

\begin{tabular}{l}
\hline \multicolumn{1}{|c|}{ AGUAS BAJAS EN ASCENSO } \\
\hline CIÉNAGAS PREDOMINIO DE LAS ESPECIES UInaria lanceolata, \\
PRagilaria capucina, Lymbia cf limnetica, Y Achanthes inflata . \\
CANNOS \\
PREDOMINIO DE LAS ESPECIES Fragilaria capucina, \\
Gomphonema parvulum, Achnanthidium minutissimum Y \\
Nitzchia palea. \\
\hline
\end{tabular}
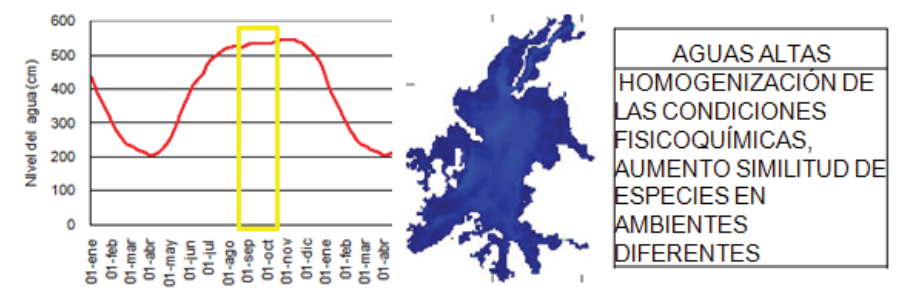

\begin{tabular}{l}
\hline \multicolumn{1}{|c|}{ AGUAS ALTAS } \\
\hline CIÉNAGAS \\
PREDOMINIO DE LAS ESPECIES Fragilaria capucina, \\
Euastrum cf ansatum, Eunotía naegueli Y Lymbia cf limnetica. \\
CAÑOS \\
PREDOMINIO DE LAS ESPECIES Lymbia cf limnetica, \\
Fragilaria capucina Y Planothidium lanceolata \\
\hline
\end{tabular}

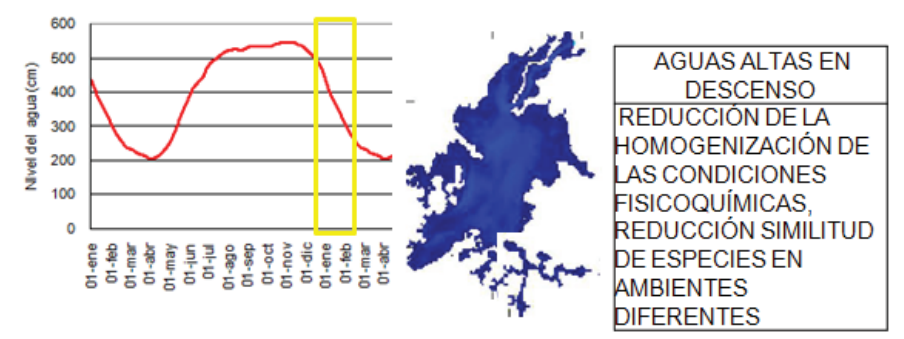

\begin{tabular}{|l|}
\hline \multicolumn{1}{|c|}{ AGUAS ALTAS EN DESCENSO } \\
\hline CIENAGAS \\
PREDOMINIO DE LAS ESPECIES Eunotía naegueli, \\
Diadesmis confervaceae, Stenopterobia delicatissima, \\
Spondiosilum rectangulare, Mougeotia sp, Navicula aff \\
rostellata, Synedra goulardii Y Brachysira cf. manfredii . \\
CAÑOS \\
PREDOMINIO DE LAS ESPECIES Lymbia cf limnetica, \\
Fragilaria capucina Y Planothidium lanceolata \\
\hline
\end{tabular}

Figura 2. Análisis general de las especies ficoperifíticas en las ciénagas y los caños, a través de las fases del pulso de inundación.

caudal del río y caños (efecto del pulso fase de desecación). Dinámica particular de las diferentes variables físico-químicas en los caños y en las ciénagas, debido al aislamiento (o desconexión) con el río. Ello determinó que las estructuras de los ensambles epífitos fueran diferentes de un cuerpo de agua a otro. Predominan en las ciénagas las especies Planothidium lanceolatum, Encyonema jetlandicum y Oscillatoria sp.1 y en los caños Navicula cryptocephala, Stenopterobia delicatissima y Eunotia naegueli.

2) Aguas Bajas en Ascenso. Reducción del efecto de cada sistema sobre el ensamble e incremento del efecto del pulso de inundación. En las ciénagas predominaron las especies Ulnaria lanceolata, Fragilaria capucina y Lyngbya cf. limnetica. En los caños fueron abundantes F. capucina, Achnanthes inflata, Achnanthidium minutissimum, Gomphonema paruulum y Nitzschia palea.

3) Aguas Altas. Uniformidad de las condiciones fisicoquímicas en los caños y en las ciénagas por la entrada de agua desde el río. Similitud de la composición específica del ficoperifiton en los diferentes ambientes. Predominan en las ciénagas $F$. capucina, Eunotia naegueli, Euastrum cf. ansatum, y L. cf. limnetica y en los caños F. capucina, P. lanceolatum y Lyngbya cf. limnetica.

4) Aguas Altas en Descenso. Reducción de la uniformidad en las condiciones fisicoquímicas del agua, por la disminución del caudal procedente del río. Reducción en la similitud de la composición específica de los ensambles de diferentes ambientes. Predominaron en las ciénagas, las especies Synedra goulardii, E. naegueli, Diadesmis confervaceae, Brachysira cf. manfredii Navicula rostellata, S. delicatissima, Spondylosium rectangulare y Mougeotia sp. En los caños fueron abundantes $L$. cf. limnetica, $F$. capucina y $P$. lanceolatum.

Conflictos de intereses: El manuscrito fue preparado y revisado con la participación de todos los autores, quienes declaramos que no existe ningún conflicto de intereses que 
ponga en riesgo la validez de los resultados presentados. Financiación: Este estudio fue financiado por la Universidad de Antioquia, el grupo de investigación Gaia y por el convenio Universidad de Antioquia-Gaia-Universidad Nacional de Colombia, en el proyecto Grecia-Colciencias.

\section{BIBLIOGRAFÍA}

1. ALLAN, J. 1995. Stream Ecology. Chapman y Hall, Londres. 388p.

2. APHA, AWWA, WPCF. 2005. Standard methods for the examination of water and wastewater. American Public Health Association (APHA), American Water Works Association (AWWA) and Water Environment Federation (WEF) (Washington). 1325p.

3. BORCHARDT, M.A. 1996. Nutrients. In Stevenson, R.J.; Bothwell, M.L.; Lowe, R.L. (eds) Algal Ecology. Freshwater benthics ecosistems. Academic Press, INC. San Diego, California, p.184-218.

4. CONDE, D.S.; BONILLA, L.; AUBRIOT R, DE LEÓN.; PINTOS, W. 1999 Comparison of the areal amount of chlorophyll a of planktonic and attached microalgae in a shallow coastal lagoon. Hydrobiol. 408409:285-291.

5. COESEL, P.F. 1996. Biogeography of desmids. Hydrobiol. 336:41-53.

6. COESEL, P.F. 1982. Structural characteristics and adaptations of desmid communities. J. Ecol. 70:163-177.

7. DONATO, R.J. 2001. Fitoplancton de los lagos andinos del Norte de Suramérica (Colombia): Composición y Factores. Academia Colombiana de Ciencias Exactas, Físicas y Naturales. Colección Jorge Álvarez Lleras $\mathrm{N}^{\circ} 19$. Bogotá, 231p.

8. FELISBERTO, S.A.; RODRIGUES, L.; LEANDRINI, J.A. 2001. Chloroccoccales registradas en la comunidad perifítica de la represa Corumbá, Estado de Goiás, Brasil, antes y después del represamiento de las aguas. Acta Scient. 23(2):275-282.

9. GOLDSBOROUGH, L.G.; ROBINSON, G.G. 1996. Pattern in wetlands. In Algal ecology, freshwater benthic ecosystems (Stevenson, R.J.; Bothwell, M.L.; Lowe, R.L. (eds.). Academic Press, San Diego. p.78-117.

10. HERNÁNDEZ-ATILANO, E.; AGUIRRE, N.J.; PALACIO, J.A. 2005. Variación espacio-temporal de la estructura de la comunidad de algas perifíticas en la mi- crocuenca de la quebrada la vega, municipio de San Roque (Antioquia), Colombia. Act. Biol. 27(82):6777.

11. HORNER, R.R.; WELCH, E.B.; SEELEY, M.R.; JACOBY, J.M. 1990. Responses of periphyton to changes in current velocity, suspended sediment and phosphorus concentration: Freshwater Biol. 24(2):215-232.

12. HUSZAR, V.L.M.; SILVA, L.H.S.; MARINHO, M.; DOMINGOS, P.; SANT'ANNA, C.L. 2000. Cyanoprokaryote assemblages in eight productive tropical Brazilian waters. Hydrobiol. 424:67-77.

13. INSTITUTO GEOGRÁFICO AGUSTIN CODAZZI (IGAC). 1986. Estudio general de los suelos de los municipios de Ayapel, Buenavista, Planeta Rica y Pueblo Rico (Departamento de Córdoba). Bogotá. 282p.

14. JUNK, W.J.; BAYLEY, P.B.; SPARKS, R.E. 1989. The flood pulse concept in river -floodplain systems. En: Dodge, D.P. (ed). Proceedings of the international large river symposium. Can. Sper. Publ. Fish. Aquat. Sci. 106:110-127.

15. MAJEWSKI-ALGARTE, V.; MORESCO, C.; RODRIGUES, L. 2006. Algas do perifíton de distintos ambientes na planície de inundação do alto rio Paraná. Acta Sci. Biol. Sci. 28(3):243-251.

16. MARTÍNEZ, L.F.; DONATO, J. 2003. Efectos del caudal sobre la colonización de algas en un río de alta montaña tropical (Boyacá, Colombia). Caldasia 25(2):337-354.

17. MONTOYA-MORENO, Y.; RAMÍREZ, J.J. 2007. Variación estructural de la comunidad perifítica colonizadora de sustratos artificiales en la zona de ritral del río Medellín, Colombia. Rev. Biol. Trop. 55(2):585-593.

18. MONTOYA-MORENO, Y.; AGUIRRE, N. 2008. Asociación de algas perifíticas en raíces de macrófitas en una ciénaga tropical Colombiana. Hidrobiol. 18(3):189198.

19. MONTOYA-MORENO, Y.; AGUIRRE, N. 2009. Dinámica del perifiton asociado con macrofitas en la ciénaga de Escobillitas y su relación con el pulso de inundación. Rev. U. Tecnol. Chocó. 28(2):196-202.

20. MONTOYA-MORENO, Y.; RAMÍREZ, J.J. 2009. Diatomeas perifíticas de la zona de ritral del río Medellín, Antioquia (Colombia). Rev. Act. Biol. 30(89):181-189. 
21. MONTOYA-MORENO, Y.; AGUIRRE, N. 2010. Dinámica de la producción primaria fitoplanctónica en un lago tropical (ciénaga Escobillitas) a lo largo del pulso de inundación. Rev. Fac. Ingeniería. U. Antioquia. 55(2):76-89.

22. MONTOYA-MORENO, Y.; AGUIRRE, N.; GONZÁLEZ, E.M. 2011. El perifiton del sistema cenagoso de Ayapel, Córdoba-Colombia. Editorial Universidad de Medellín. ISBN: 978-958-8692-42-5. 84p.

23. MURPHY, K.J.; DICKINSON, G.; THOMAZ, S.M.; BINI, L.M.; DICK, K.; GREAVES, K.; KENNEDY, M.P.; LIVINGSTONE, S.; MCFERRAN, H.; MILNE, J.M.; OLDROYD, J.; WINGFIELD, R.A. 2003. Aquatic plant communities and predictors of diversity in a subtropical river floodplain: the upper Rio Paraná, Brazil. Aqu. Bot. 77:257-276.

24. NEIFF, J.J. 1999. El régimen de pulsos en ríos y grandes humedales de Sudamérica. 97-145. En: Malvarez, A.I.; Kandus, P. (eds.). Tópicos sobre grandes humedales sudamericanos. ORCYT-MAB-UNESCO. p. 224.

25. RAMÍREZ, A.M.; PLATA-DÍAZ, Y. 2008. Diatomeas perifíticas en diferentes tramos de dos sistemas lóticos de alta montaña (Páramo de Santurbán, norte de Santander, Colombia) y su relación con las variables ambientales. Acta Biol. Col. 13(1):199-216.

26. RIVERA-RONDÓN, C.A.; DÍAZ, C. 2004. Grandes taxones de fitobentos y su relación con la hidrología, física y química de pequeños ríos andinos. Universitas Scientiarum. 9:75-86.

27. RIVERA-RONDÓN, C.A.; ZAPATA, A.M.; PÉREZ, D.; MORALES, Y.; OVALLE, H.; ÁLVAREZ, J.P. 2010. Caracterización limnológica de humedales de la planicie de inundación del río Orinoco (Orinoquía, Colombia). Acta Biol. Col. 15(1):1-16.

28. RODRIGUES, L.; BICUDO, D.C. 2001. Similarity among periphyton algal communities in lentic-lotic gradient of the upper Paraná river floodplain, Brazil. Rev. Bras. Bot. 24(3):235-248.
29. RODRIGUES, L.; ZANON, J.E.; CARAPURNALA, L.; BIOLO, S. 2008. Perifiton. En: A Planície Alagável do Rio Paraná: Estrutura e Processo Ambiental. Disponible desde Internet en http://www.peld.uem.br/Relat2008/ pdf/Capitulo04.pdf (con acceso el 17/07/2010).

30. ROLDÁN, P.G.; RAMÍREZ, J.J. 2008. Fundamentos de limnología neotropical. Universidad de Antioquia,

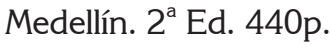

31. ROSS, J. 1979. Prácticas de ecología. Omega, Barcelona, España. 250p.

32. SCHWARZBOLD, A. 1990. Mêtodos ecológicos aplicados ao estudo do perifiton. Acta Limn. Bras. 3:545592.

33. SCHINDLER, D.W. 1977. Evolution of phosphorus limitation in lakes. Science. 195:260-262.

34. STEVENSON, R.J. 1996. The Stimulation and Drag of Current. En: Algal Ecology Freshwater Benthic Ecosystems. Stevenson, R.J., Bothwell, M.L.; Lowe, R.L. (eds). Academic Press. p.141-168.

35. TANIGUCHI, G.M.; BICUDO, D.C.; SENNA, P.A. 2005. Gradiente litorâneo-limnético do fitoplâncton e ficoperifiton em uma lagoa da planicie de inundacâo do Rio Mogi-Guaḉu. Rev. Bras. Bot. 28(1):137-147.

36. THOMAZ, S.M.; BINI, L.M.; BOZELLI, R.L. 2007. Floods increase similarity among habitats in river-floodplain systems. Hydrobiol. 579:1-13.

37. WENZEL, M.T.; DÍAZ, M.M. 2008. Cyanophyta del parque nacional Nahuel Huapi (Argentina), II. Darwiniana. 46(1):51-65.

38. ZAPATA, A.M.; DONATO, R.J. 2005. Cambios diarios de las algas perifíticas y su relación con la velocidad de la corriente en un río tropical de montaña (río TotaColombia). Caldasia. 24(3-4):327-338.

Recibido: Diciembre 21 de 2012

Aceptado: Julio 13 de 2013

Como citar:

Montoya-Moreno, Y.; Aguirre, N. 2013. Dinámica del ensamblaje algal epifítico en el sistema de planos inundables de Ayapel a través del pulso de inundación. Rev. U.D.CA Act. \& Div. Cient. 16(2): 491-500. 